\section{Thalidomide for the treatment of gastrointestinal bleeding due to angiodysplasia in a patient with Glanzmann's thrombasthenia}

\author{
Bruno K.L Duarte, ${ }^{1}$ Sílvia M. de Souza, \\ Carolina Costa-Lima, ${ }^{1}$ \\ Samuel S. Medina, ${ }^{1}$ \\ Margareth C. Ozelo ${ }^{1,2}$ \\ 1Instituto Nacional de Ciência e \\ Tecnologia do Sangue, Hemocentro \\ Unicamp, and ${ }^{2}$ Department of Internal \\ Medicine, School of Medical Sciences, \\ University of Campinas, Campinas, SP, \\ Brazil
}

\begin{abstract}
Angiodysplasia is a frequent cause of persistent gastrointestinal (GI) hemorrhage in elderly patients. Although GI bleeding isn't the most common manifestation in patients with bleeding disorders, when present, it represents a challenging complication. We describe a 62-year-old patient with Glanzmann's thrombasthenia, who used thalidomide for severe and recurrent GI bleeding. For 6 months, the patient experienced temporary control of GI bleeding with thalidomide in a daily oral dose of 100 $\mathrm{mg}$. The anti-angiogenic effects of thalidomide have recently been explored by several groups, particularly in the management of bleeding from angiodysplasia, including cases with von Willebrand disease. Here, we review the relevant descriptions of the use of thalidomide in this situation, and also discuss potential reasons why we observed only a temporary control of the GI bleeding in our patient, such as the use of low-dose regimen due to limitations posed by thalidomide side effects.
\end{abstract}

\section{Introduction}

Bleeding manifestations in patients with inherited bleeding disorders can be diverse. ${ }^{1}$ Although gastrointestinal (GI) hemorrhage is not one of the most common bleeding manifestations in these patients, ${ }^{1}$ its occurrence may be challenging in this scenario with existing coagulopathy augmenting the bleeding intensity, increasing transfusion requirements and limiting the performance of invasive diagnostic and therapeutic endoscopic procedures.

Angiodysplastic lesions are a recurrent cause of GI hemorrhage, representing the most common cause of occult GI bleeding. ${ }^{2}$ It is an acquired disease and has been considered an elderly disease, ${ }^{3}$ since most of the lesions are detected in patients over 60 years old. The angiodysplasia occurrence is increased in patients with end-stage renal disease, congenital or acquired von Willebrand disease (vWD), and possibly aortic stenosis. The pathogenesis is uncertain, and is probably related by degenerative processes associated with aging. ${ }^{2}$ Available therapeutic approaches include endoscopic procedures, trans catheter intervention by angiography, surgery and medical therapies. ${ }^{4}$ Recently, the use of drugs with anti-vascular endothelial growth factor (VEGF) effect such as thalidomide has been reported as an option for patients with refractory gastrointestinal bleeding secondary to angiodysplasias. ${ }^{4-7}$

Glanzmann's Thrombasthenia (GT) is an autosomal recessive platelet surface receptor disorder of the glycoprotein (GP) $\mathrm{IIb} / \mathrm{III}$. The bleeding phenotype is variable. However, the majority of patients will require platelet transfusion during their lifetime. Platelet refractoriness due to the presence of isoantibodies against GPIIb/IIIa and/or human leukocyte antigens (HLA) is a frequent complication for the GT patients undergoing repeated platelet transfusion. Currently, recombinant activated factor VII concentrates (rFVIIa) have been successfully used to control the bleeding symptoms of these patients. ${ }^{8}$ Here, we report the case of a female patient who was diagnosed with GT at the age of 15 and presented with persistent GI bleeding in her 60s.

\section{Case Report}

We report the case of a 62-year-old female with a familial history of bleeding disorder diagnosed with GT at the age of 15 during the investigation of menorrhagia. She had been frequently transfused throughout her life, until her menopause at 52, when bleeding episodes ceased.

The patient did not present with additional bleeding manifestations until she was 60 when the first GI bleeding episode occurred. In November 2012, she was admitted at a local hospital due to massive lower GI bleeding. She was severely anemic, requiring transfusion of many units of packed red blood cells (PRBC). Colonoscopy was performed and detected an angiodysplastic lesion at the rectum and another at the cecum with active bleeding. Adrenaline injection therapy was performed and an endoscopic clip was successfully placed and stopped the bleeding from the
Correspondence: Margareth Castro Ozelo, IHTC Haemophilia Unit Cláudio Luiz Pizzigatti Corrêa, INCT do Sangue Hemocentro Unicamp, University of Campinas, Rua Carlos Chagas 480, Cidade Universitária, Campinas, São Paulo 13.083.878 Brazil.

Tel.: +55.19.3521.8395 - Fax: +55.19 .3521 .8670 .

E-mail: margaret@unicamp.br

Key words: angiodysplasia; thalidomide; Glanzmann's thrombasthenia; gastrointestinal bleeding.

Contributions: SMS collected and wrote the manuscript; SSM and CSCL followed the patient, collected the data, and wrote the manuscript; BKLD wrote, reviewed the manuscript; MCO collected the data, and critically revised and approved the final version of the manuscript.

Conflict of interest: the authors declare no potential conflict of interest.

Funding: the authors received funding support from Fundação de Amparo à Pesquisa do Estado de São Paulo (FAPESP).

Received for publication: 10 November 2016 . Accepted for publication: 21 March 2017.

This work is licensed under a Creative Commons Attribution-NonCommercial 4.0 International License (CC BY-NC 4.0).

(C) Copyright B.K.L Duarte et al., 2017

Licensee PAGEPress, Italy

Hematology Reports 2017; 9:6961

doi:10.4081/hr.2017.6961

active lesion. At that time, she was also started on tranexamic acid. One month later she was re-evaluated in our service referring sporadic GI bleeding episodes that were controlled with the continuous use of tranexamic acid.

During the following months, she experienced several GI bleeding episodes requiring transfusion of a total of 25 units of PRBC (Figure 1). Oral and intravenous iron replacement therapy were started during this period. Three upper GI endoscopies, two colonoscopies, one capsule endoscopy and a radionuclide scan were performed on different bleeding episodes she experienced between September 2013 and August 2014 and failed to identify the origin of the bleeding.

A presumptive diagnosis of angiodysplasia was made and a trial of thalidomide was started in September 2014, on a dose of $50 \mathrm{mg}$ daily. After 15 days and no side effects reported, the dose was increased to $100 \mathrm{mg}$ daily and continued that way for almost 6 months. While on thalidomide 
treatment, she started to present drowsiness and mild paresthesia on her lower limbs. Continuous tranexamic acid and iron replacement were maintained. During the period of thalidomide use, a reduction in the number and severity of bleeding episodes as well as in transfusion requirements was noted (Figure 1). After five months on thalidomide, the patient started to present recurrent GI bleeding episodes that demanded treatment with rFVIIa, platelet transfusion, and continuous tranexamic acid. Thalidomide was then suspended. At this occasion, platelet refractoriness was confirmed with the presence of antiGPIIb/IIIa (Gi5) demonstrated by monoclonal antibody-specific immobilization of platelet antigen (MAIPA) test. Bleeding became more severe and were only partially controlled with rFVIIa and platelet transfusion. She died in April 2015, due to persistent hemorrhage and sepsis caused by hospital-acquired infection.

\section{Discussion}

Angiodysplastic lesions are a recurrent cause of hemorrhage and are the most common cause $(40 \%)$ of occult GI bleeding in the general population, especially in elderly patients. ${ }^{9}$ The association between GI bleeding due to angiodysplasia and vWD is well recognized and described by many authors. ${ }^{10-13}$ However, there is a paucity of data regarding the relationship of other rare bleeding disorders with GI bleeding from angiodysplasia.

In a recent review of bleeding assessment tools, ${ }^{1}$ GI hemorrhage was found to be the ninth cause of bleeding symptoms, with a prevalence of $14 \%$ in all types of vWD and $1 \%$ in healthy subjects.

Management of angiodysplastic bleeding is often difficult and challenging. GI angiodysplasia commonly presents with multiple lesions, that can be difficult to access and treat. Bleeding episodes are usually recurrent and severe and can cause significant morbidity. ${ }^{4}$

Despite several available diagnostic methods, angiodysplastic lesions are still extremely difficult to detect. ${ }^{14}$ Accurate diagnosis requires active bleeding at the moment of the examination, which often leaves patients undiagnosed. In our patient, although an angiodysplastic lesion was detected initially, in all subsequent episodes diagnostic methods failed to identify the source of bleeding. In a retrospective study evaluating patients with vWD and GI bleeding, ${ }^{3}$ investigation studies were negative in 30 of the $48(62.5 \%)$ patients. In the remaining $18(37.5 \%)$ cases, the cause of GI bleeding was confirmed to be angiodysplasia.

Various therapeutic options have been described to control the bleeding associated with angiodysplastic lesions. Treatment modalities encompass endoscopic therapies, trans catheter angiography and intervention, surgery and medical therapies. ${ }^{4}$ Some of the currently used pharmacological options are combined hormonal therapy (estrogen and progesterone) and the somatostatin analogue octreotide. However, evidence on their effectiveness has not yet been adequately established. For patients with vWD, the use of desmopressin (DDAVP $\AA$ ), von Willebrand factor containing products, factor concentrates and antifibrinolytic amino acids (tranexamic acid and epsilon aminocaproic acid) are also used. Nevertheless, the persistence of the angiodysplastic lesion and the underlying bleeding disorder renders them less effective. In a systematic review regarding therapies for angiodysplasia and gastric antral vascular ectasia, ${ }^{15}$ the authors found insufficient or low evidence data regarding the efficacy for current therapeutic approaches, pointing out the necessity of more effective treatment options.

Currently, the use of drugs with antiangiogenic properties in refractory GI bleeding due to angiodysplasias have been reported. Thalidomide is a drug with a potent antiangiogenic effect and acts by suppressing the expression of VEGF. It acts by both inhibiting angiogenesis and promoting vessel maturation. ${ }^{16}$ Available data on its use in this setting derives mostly from small studies and case reports of patients mainly with vWD and GI bleeding due to angiodysplasia. , $7,17-19$ Only one randomized controlled trial of thalidomide has been reported..$^{5}$ This trial enrolled 10 patients with gastrointestinal vascular malformations randomized to receive either $100 \mathrm{mg}$ of thalidomide daily or 400 $\mathrm{mg}$ of iron daily. Patients receiving thalidomide had significant increases in hemoglobin, reduction in transfusion requirements and bleeding episodes. This was, however, an open-label, single-center study with very stringent inclusion criteria and its results must be further validated. More recently, a non-randomized, phase II study of low-dose thalidomide was conducted in 31 patients with hereditary hemorrhagic telangiectasia, with the primary endpoint of epistaxis

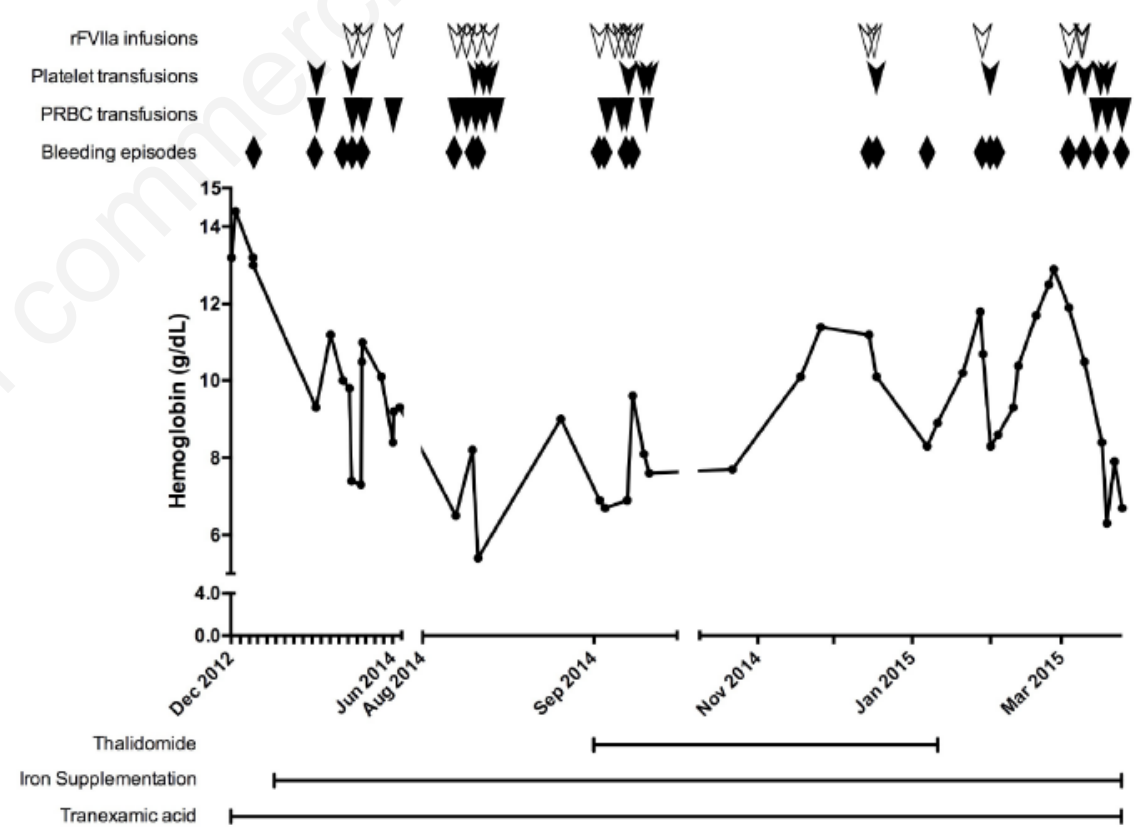

Figure 1. Patient's hemoglobin evolution during follow-up. Patient initially presented at another hospital with severe GI bleeding (data not available and not shown) in November 2012. She started follow-up at our clinic soon after, in December 2012. She developed several bleeding episodes during the following months requiring transfusion of different blood products. With the introduction of thalidomide in September 2014, a reduction in the number of bleeding episodes was observed, with no transfusion requirements during this period. In mid-January 2015, however, bleeding relapsed and she was taken of thalidomide. After placement of a peripherally inserted central venous catheter for intravenous iron infusions, she developed sepsis and died. PRBC: packed red blood cells; rFVIIa: recombinant activated factor VII. 
severity. All patients experienced a significant reduction in bleeding, with $10 \%$ showing a complete response. ${ }^{6}$

This study allowed for a dose-progression in thalidomide, up to $200 \mathrm{mg}$ daily, with $81 \%$ of patients achieving the primary endpoint with $50 \mathrm{mg}$ daily, $16 \%$ with 100 $\mathrm{mg}$ daily and $3 \%$ with $150 \mathrm{mg}$ daily. This is in accordance to recent results showing a dose-dependent inhibition of VEGF and cellular proliferation of HUVEC cultures. ${ }^{20}$ Further data from randomized controlled trials are needed to establish unequivocal benefit and optimal dosing of thalidomide in this setting.

Although limited, our patient had initially a positive response to thalidomide, with a reduction in the frequency of bleeding episodes and transfusion requirements and a low toxicity profile. This effect was, however, limited, with uncontrolled bleeding recurring after 6 months. In addition, our patient had the diagnosis of GT, an inherited platelet disorder with a common complication reported in these severe cases and refractoriness to platelet transfusion, increasing the difficulty to control bleeding manifestations.

\section{Conclusions}

In conclusion, thalidomide seems to be a potential candidate for the treatment of patients with refractory GI bleeding due to angiodysplasias. In our experience, bleeding episodes were significantly reduced but did not stop. This could be the result of using a low-dose regimen, as current data suggests the need of higher doses in some patients. In that sense, further research is needed, directed toward determining its efficacy, optimum dose and duration of treatment.

\section{References}

1. Rydz N, James PD. The evolution and value of bleeding assessment tools. J Thromb Haemost 2012;10:2223-9.

2. Foutch PG. Angiodysplasia of the gastrointestinal tract. Am J Gastroenterol 1993;88:807-18.

3. Makris M, Federici AB, Mannucci PM, et al. The natural history of occult or angiodysplastic gastrointestinal bleeding in von Willebrand disease. Haemophilia 2015;21:338-42.

4. Sami SS, Al-Araji SA, Ragunath K. Review article: gastrointestinal angiodysplasia - pathogenesis, diagnosis and management. Aliment Pharmacol Ther 2014;39:15-34.

5. Ge ZZ, Chen HM, Gao YJ, et al. Efficacy of thalidomide for refractory gastrointestinal bleeding from vascular malformation. Gastroenterology 2011;141:e1-4.

6. Invernizzi R, Quaglia F, Klersy C, et al. Efficacy and safety of thalidomide for the treatment of severe recurrent epistaxis in hereditary haemorrhagic telangiectasia: results of a non-randomised, single-centre, phase 2 study. Lancet Haematol 2015;2:e465-73.

7. Engelen ET, van Galen KP, Schutgens RE. Thalidomide for treatment of gastrointestinal bleedings due to angiodysplasia: a case report in acquired von Willebrand syndrome and review of the literature. Haemophilia 2015;21:41929.

8. Di Minno G, Coppola A, Di Minno MN, Poon MC. Glanzmann's thrombasthenia (defective platelet integrin alphaIIb-beta3): proposals for management between evidence and open issues. Thromb Haemost 2009;102:1157-64.

9. Sanchez-Capilla AD, De La TorreRubio P, Redondo-Cerezo E. New insights to occult gastrointestinal bleeding: From pathophysiology to therapeutics. World J Gastrointest Pathophysiol 2014;5:271-83.

10. Randi AM, Laffan MA, Starke RD. Von Willebrand factor, angiodysplasia and angiogenesis. Mediterr J Hematol Infect
Dis 2013;5:e2013060.

11. Franchini M, Mannucci PM. Von Willebrand disease-associated angiodysplasia: a few answers, still many questions. $\mathrm{Br} \mathrm{J}$ Haematol 2013;161:177-82.

12. Franchini M, Mannucci PM. Gastrointestinal angiodysplasia and bleeding in von Willebrand disease. Thromb Haemost 2014;112:427-31.

13. Makris M. Gastrointestinal bleeding in von Willebrand disease. Thromb Res 2006;118:S13-7.

14. Siragusa S, Malato A, Lo Coco L, et al. Gastrointestinal bleeding due to angiodysplasia in patients with type 1 von Willebrand disease: report on association and management. Haemophilia 2008; $14: 150-2$.

15. Swanson E, Mahgoub A, MacDonald R, Shaukat A. Medical and endoscopic therapies for angiodysplasia and gastric antral vascular ectasia: a systematic review. Clin Gastroenterol Hepatol 2014;12:571-82.

16. Franchini M, Frattini F, Crestani S, Bonfanti C. Novel treatments for epistaxis in hereditary hemorrhagic telangiectasia: a systematic review of the clinical experience with thalidomide. J Thromb Thrombolysis 2013;36:355-7.

17. Hirri HM, Green PJ, Lindsay J. Von Willebrand's disease and angiodysplasia treated with thalidomide. Haemophilia 2006;12:285-6.

18. Garrido A, Sayago M, Lopez J, et al. Thalidomide in refractory bleeding due to gastrointestinal angiodysplasias. Rev Esp Enferm Dig 2012;104:69-71.

19. Heidt J, Langers AM, van der Meer FJ, Brouwer RE. Thalidomide as treatment for digestive tract angiodysplasias. Neth J Med 2006;64:425-8.

20. Tan H, Chen H, Xu C, et al. Role of vascular endothelial growth factor in angiodysplasia: an interventional study with thalidomide. J Gastroenterol Hepatol 2012;27:1094-101. 